\title{
AUTOMORPHISMS OF SEMIGROUPS OF COMPLEXES OF ABELIAN GROUPS
}

BY RICHARD D. BYRD, JUSTIN T. LLOYD,

FRANKLIN D. PEDERSEN, AND JAMES W. STEPP

Communicated by Alston S. Householder, September 19, 1976

In the study of any algebraic system, one of the first objects investigated is its automorphism group [1]. The automorphisms of groups have been investigated so extensively that we make no attempt at a list of references. In [4], A. R. Richardson studied the automorphisms of groupoids. Semigroups of finite complexes in groups are central to the study of retractable groups [2], and here we announce some properties of the automorphism group of such a semigroup in the case in which the underlying group is abelian. In particular, when the underlying group is cyclic we classify these automorphism groups.

If $G$ is a group, then the collection $F(G)$ of all finite nonempty subsets of $G$ is a semigroup, where $A B=\{a b \mid a \in A$ and $b \in B\}$. Each automorphism $\alpha$ of $G$ induces an automorphism $\alpha^{*}$ of $F(G)$ where $A \alpha^{*}=\{a \alpha \mid a \in A\}$. An automorphism of $F(G)$ of this type will be called a standard automorphism of $F(G)$.

THeOREM 1. If $\varphi$ is an automorphism of $F(G)$, then $\varphi$ is a standard automorphism if and only if $\varphi$ is inclusion preserving.

A homomorphism $\sigma$ of $F(G)$ into $G$ such that $\{g\} \sigma=g$ for every $g$ in $G$ is called a retraction of $G$. A group $G$ is called retractable if it admits a retraction. The concept of a retractable group was introduced in [2] where it was shown that the class of retractable groups is a proper subclass of the class of torsion free groups and the class of lattice-ordered groups is a proper subclass of the class of retractable groups. Hence, the class of torsion free abelian groups is a proper subclass of the class of retractable groups. "It seems to be a rather difficult problem to determine all abelian groups with commutative endomorphism ring" $[3$, p. 205]. If $G$ is a torsion free abelian group then it is easy to show that the automorphism group of $F(G)$ is nonabelian.

THEOREM 2. If $G$ is an abelian group, $\sigma$ is a retraction of $G$, and $\varphi_{\sigma}$ is given by

$$
A \varphi_{\sigma}=(A \sigma) A\left(A^{-1} \sigma\right)
$$

for every $A \in F(G)$, then $\varphi_{\sigma}$ is an automorphism of $F(G)$. Moreover,

(i) if $\varphi_{\sigma}$ is not the identity automorphism, then $\varphi_{\sigma}$ is a nonstandard automorphism of $F(G)$ of infinite order;

AMS (MOS) subject classifications (1970). Primary 20M15. 
(ii) the automorphism group of $F(G)$ is an infinite nonabelian group.

Let $Z$ denote the additive group of integers. In [2] the collection of retractions of $Z$ was completely determined. If $k \in Z$ and if $\sigma_{k}$ is defined by $A \sigma_{k}=(k+1) \max A-k \min A$ for all $A \in F(Z)$, then $\left\{\sigma_{k} \mid k \in Z\right\}$ is the collection of retractions of $Z$. We have shown that the automorphisms of $F(Z)$ that are induced by retractions of $Z$ are contained in the cyclic subgroup generated by $\varphi_{\sigma_{-1}}$. With this information we are able to show

THEOREM 3. The automorphism group of $F(Z)$ is isomorphic to a nonabelian splitting extension of the integers by the Klein four-group.

If $n$ is a natural number, let $Z_{n}$ denote the group of integers modulo $n$. Clearly, the automorphism groups of $F\left(Z_{1}\right)$ and $F\left(Z_{2}\right)$ are trivial. It can be shown that $F\left(Z_{3}\right), F\left(Z_{4}\right)$, and $F\left(Z_{5}\right)$ admit nonstandard automorphisms.

THEOREM 4. If $n$ is a natural number, $n \geqslant 6$, then $F\left(Z_{n}\right)$ admits only standard automorphisms and hence the automorphism group of $F\left(Z_{n}\right)$ is isomorphic to the group of automorphisms of $Z_{n}$.

The proofs of the above results, as well as others, are computational and will appear elsewhere.

\section{BIBLIOGRAPHY}

1. N. Biggs, Finite groups of automorphisms, Cambridge Univ. Press, New York and London, 1971. MR 48 \#5905.

2. R. D. Byrd, J. T. Lloyd, R. A. Mena and J. R. Teller, Retractable groups, Acta. Math. Acad. Sci. Hungar. (to appear).

3. L. Fuchs, Abelian groups, Pergamon Press Ltd., New York 1960 . MR 22 \#2644.

4. A. R. Richardson, Groupoids and their automorphisms, Proc. London Math. Soc. (2) 48 (1943), 83-111. MR 5, 60.

DEPARTMENT OF MATHEMATICS, UNIVERSITY OF HOUSTON, HOUSTON, TEXAS 77004

DEPARTMENT OF MATHEMATICS, SOUTHERN ILLINOIS UNIVERSITY, CARBONDALE, ILLINOIS 62901 